\title{
Complex angular momenta approach for scattering problems in the presence of both monopoles and short range potentials
}

\author{
Fabrizio Canfora ${ }^{1}$ \\ ${ }^{1}$ Centro de Estudios Científicos (CECS), Casilla 1469, Valdivia, Chile. \\ canfora@cecs.cl
}

October 4, 2018

\begin{abstract}
It is analyzed the quantum mechanical scattering off a topological defect (such as a Dirac monopole) as well as a Yukawa-like potential(s) representing the typical effects of strong interactions. This system, due to the presence of a short-range potential, can be analyzed using the powerful technique of the complex angular momenta which, so far, has not been employed in the presence of monopoles (nor of other topological solitons). Due to the fact that spatial spherical symmetry is achieved only up to internal rotations, the partial wave expansion becomes very similar to the Jacob-Wick helicity amplitudes for particles with spin. However, since the angular-momentum operator has an extra "internal" contribution, fixed cuts in the complex angular momentum plane appear. Correspondingly, the background integral in the Regge formula does not decrease for large values of $|\cos \theta|$ (namely, large values of the Mandelstam variable $s$ ). Hence, the experimental observation of this kind of behavior could be a direct signal of non-trivial topological structures in strong interactions. The possible relations of these results with the soft Pomeron are shortly analyzed.
\end{abstract}




\section{Introduction}

One of the most important non-perturbative effects in Field Theory is the appearance of topological solitons [1] [2] [3] [4]. Such classical configurations are topologically stable and, for this reason, are believed to play a crucial role at a quantum level as well (the most important arena being, of course, the problem of color confinement: for a pedagogical review on the role of topological configurations in the problem of confinement see [5]). Such objects possess a conserved topological charge which prevents them from being deformed to the trivial vacuum. A key characteristic of such non-trivial topological structures is that, due to the non-vanishing topological charge, spatial spherical symmetry must be realized in a rather subtle way. As it was firstly realized in modern terms by Skyrme when introducing his famous model [6] (but the same argument also applies when dealing with local internal symmetries), the most obvious way to describe a spherical object would be to take all the fields of the Lagrangian to depend only on the radial coordinate. However, in this case the topological charge would vanish (as an easy computation shows immediately). Thus, the most one can get is to have the fields depending on the angular coordinates as well but in such a way that energy density is spherically symmetric. This, actually, is the definition of hedgehog ansatz: the hedgehog is symmetric under spatial rotations but only up to an internal symmetry transformation (namely, the spatial rotation needs to be compensated by an internal symmetry transformation in order to achieve the sought invariance). This quite simple characteristic is behind the remarkable intuition of Skyrme that his hedgehog (called Skyrmion), despite being constructed in a purely Bosonic theory, should be actually quantized as a Fermion. The reason is that the generators of the angular momentum acquire extra contributions from the internal symmetry transformation so that the eigenvalues are not required to be integer anymore and can be also half-integers.

The simplest and most famous example in gauge field theory which gives rise to similar phenomena is the Dirac monopole [7]. In the case of the Dirac monopole too one gets spherical symmetry up to an internal (gauge) symmetry transformation. This fact has highly non-trivial consequences when one analyzes the Schrodinger (as well as the Klein-Gordon and Dirac) equation(s) in the electromagnetic field 1 of a Dirac monopole. It was soon realized that the angular momentum operator in the case of the Schrodinger equation in the field of a Dirac monopole (which was studied for the first time in [8] and [9]) receives an extra contribution and that such modified angular momentum operator is responsible for the fact that a scalar charged particle in the field of a Dirac monopole can behave as a Fermion (for a very clear analysis see [10]). Such non-trivial feature of the total angular momentum of a monopole is responsible of remarkable effects not only in Quantum Field Theory (QFT henceforth) as already emphasized but also in general relativity (see, in particular, [11]).

A very far-reaching generalization of the Dirac monopole has been constructed in non-Abelian gauge theory coupled to a Higgs field in the adjoint representation of the gauge group by ' $t$ Hooft and Polyakov [12] 13]. Such configurations are in a sense a regularized version of the Dirac monopole

\footnotetext{
${ }^{1}$ Here and in the following sections the sentence "the Schrodinger (Dirac, Klein-Gordon) equation(s) in the electromagnetic field of a Dirac monopole" will always mean the Schrodinger (Dirac, Klein-Gordon) equation(s) with the minimal coupling $\partial_{\mu} \rightarrow \nabla_{\mu}=\partial_{\mu}-i e A_{\mu}$ to the gauge potential $A_{\mu}$ of a Dirac monopole.
} 
as, far from the monopole core, they look like Dirac monopoles while having, at the same time, regular cores (for a detailed review, see [1] [3]). Also non-Abelian monopoles are hedgehog in the sense that the corresponding gauge fields depend both on the radial coordinate and on the angles but in such a way that the topological charge (which in this case represents the non-Abelian magnetic charge) is non-vanishing and, at the same time, the energy-density is spherically symmetric. For this reason, the generators of the angular momentum acquire extra contributions from the generator of the gauge symmetry. Hence, once again, the eigenvalues are not required to be integers anymore and can be also half-integers. This fact is at the basis of the well-known 't Hooft-Hasenfratz-Jackiw-RebbiGoldhaber phenomenon [14] [15] [16] which states that a scalar field charged under the gauge group is transformed into a Fermion in the field of a 't Hooft-Polyakov monopole. Moreover, this effect persists even if the effects of General Relativity are turned on [17]. The 't Hooft-Polyakov monopole is not known analytically in the realistic case 2 in which the Higgs potential is non-vanishing. In this case, far from the core, one expects Yukawa-like behavior of the Higgs fields together with the massless behavior of the gauge field associated with the unbroken $U(1)$ gauge symmetry (which, asymptotically, looks exactly like a Dirac monopole). Consequently, a very interesting analysis is the study of scattering from both Dirac monopole and Yukawa's potentials.

The above considerations show how crucial are the modified generator of the angular momentum operator in the analysis of the physical properties of topologically non-trivial configurations.

The most obvious and natural way to probe (both theoretically and experimentally) this kind of objects is of course through scattering [20] [21] [22]. Due to the interest in this non-trivial configurations, the scattering of charged particles on monopoles and dyons has been deeply analyzed: very important references in this respect (after the pioneering works [8] and [9]) are [23] [24] [25] [26]. One of the most important technical results (which will be very useful in the following) achieved in these references has been the proper definition of the partial wave expansion in the presence of a monopole. However, what was not considered in these references is the inclusion of a (superposition of) Yukawa's potential(s): as it is clear from the above considerations, such an inclusion is very natural and welcome from the point of view of non-Abelian monopoles. Moreover, from a QFT perspective (see, for instance, [27]) when dealing with strong interactions, it is very natural to expect the presence of (superpositions of) Yukawa's potentials. Thus, a very natural problem to consider (which, to the best of author's knowledge, has not been analyzed before) is the quantum mechanical scattering both from a monopole and (a superposition of) Yukawa's potential(s) which, at the very least, describes the scattering processes far from the core of a 't Hooft-Polyakov monopole.

At a first glance, one could think that such a problem is technically much more complex than the original analysis of [23] 24] 25] 26] as one cannot get neither the wave function nor the spectrum in closed forms. However, a huge benefit (which, in the opinion of the present author, vastly exceeds the above mentioned technical disadvantages) of the presence of Yukawa potentials is that very powerful tools become available: the Sommerfeld-Watson transform and the Regge theory of complex angular momenta [28] [29] (which cannot be applied directly to the scattering from monopoles or dyons alone

\footnotetext{
${ }^{2}$ Exact solutions for the non-Abelian monopoles can be found in the BPS approximation [18] 19] in which the Higgs potential vanishes but one keeps the non-trivial boundary conditions.
} 
mainly because of the long-range effects of the monopoles). The applications of such techniques (which have been extended to quantum field theory in [30, 31, 36] [32] 333 [34; detailed reviews are [37] and [38]) in the context of strong interactions together with the Mandelstam representation [35] have been extremely successful. Moreover, due to the fundamental role of the angular momentum within the Regge approach, it is clear that such an approach is very suitable when dealing with scattering from topologically non-trivial objects which are characterized (as it has been discussed above) by modified angular momentum operators. For instance, a very interesting by-product of the present analysis is that it disclose in a very clear way the similarities (which should be expected, from the intuitive point of view, on the basis of [10]) between the helicity amplitudes for particles with spin introduced by Jacob and Wick in their pioneering paper [39] and the partial-wave expansion in the presence of both monopoles and short range potential. However, there is also a crucial difference between the two partial wave amplitudes which could be interpreted as a fingerprint of the presence of non-trivial topological structures in gauge theories as it will be discussed in the next sections.

Besides the intrinsic theoretical interest of this analysis, the application of the theory of complex angular momenta when topological solitons are present could be relevant for a very well known open problem in the field of strong interactions: the Pomeron [40] (detailed reviews are [41] [42]). The key issue is that at low transferred momentum $t$ observations show that the scattering amplitude does not decrease with $s$ as one would expect on the basis of the Froissart-Martin bound [36] [43] (a modern and interesting analysis can be found in [44]). It is worth emphasizing that the Froissart-Martin bound can be derived from very general hypothesis such as unitarity, analyticity as well as from the short range nature of strong interactions. The present analysis shows that the hypothesis of short range interactions is rather subtle when topological defects are present. Indeed, one of the typical contributions in the Schrodinger (as well as Klein-Gordon) equation(s) when a monopole is present is that the centrifugal barrier increases. This implies, in particular, that the effects of the monopole are not short-range as one can feel them even from very far. There are many approach to the Pomeron based on QCD, the most powerful being the BFKL equation [45] [46] [47] (detailed review are [48] [49]). However, the issue related to the low $t$ behavior is still open. As it will be explained in the next sections, the present analysis suggests that the inclusion of the non-trivial topological structures of the Yang-Mills vacuum within the BFKL formalism could be an important step to solve this issue.

As far as the increase of the centrifugal barrier induced by topological solitons is concerned, it is worth emphasizing 3 that, in $2+1$ dimensions, a similar effect does occur as well. In particular, the Chern-Simons term describing the interaction of a charge with the $(2+1)$-dimensional magnetic monopole (see, for instance, the detailed review [50]) manifests itself by changing the plane geometry felt by a free particle into a $(2+1)$-dimensional geometry with a conical defect. Not surprisingly, at classical level a charged particle moving into the electromagnetic field generated by a Dirac monopole in $(3+1)$ dimensions also moves along conical surfaces (see [51] and, for a detailed review, the first two chapters of [3]).

The paper is organized as follows: in the second section, there is a short review of the usual Watson-

\footnotetext{
${ }^{3}$ I thank the anonymous referee for this interesting remark.
} 
Sommerfeld-Regge transform. In the third section, the Schrodinger equation in the presence of both a monopole and a short range together with the corresponding scattering amplitude are discussed. In the fourth section, the Watson-Sommerfeld-Regge transformation in the presence of monopoles and short range potential is analyzed. In the fifth section, the limitations of the present approach are presented. In the sixth section, the relativistic generalization of the results are introduced and the relations with the BFKL equation are shortly emphasized. Finally, in the last section some conclusions and perspectives are included.

\section{Review of Watson-Sommerfeld-Regge transform}

Here all the necessary ingredients needed to perform the Watson-Sommerfeld-Regge transform in quantum mechanical scattering are shortly reviewed with particular emphasis on the technical steps which are going to change in the presence of topological solitons. Let us begin with the usual expression for the partial wave expansion of the scattering amplitude:

$$
\begin{aligned}
f(\theta, k) & =\frac{1}{2 k i} \sum_{l=0}^{+\infty}(2 l+1) P_{l}(\cos \theta)\left[\exp \left(2 i \delta_{l}(k)\right)-1\right], E=\frac{k^{2}}{2 M}, \\
k^{2} \psi & =\left[-\frac{d^{2}}{d r^{2}}+\frac{\lambda^{2}-\frac{1}{4}}{r^{2}}+V\right] \psi, \\
\lambda & =l+\frac{1}{2}, \\
V(r) & =\int_{M_{0}}^{\infty} \sigma(\rho) \frac{\exp [-\rho r]}{r} d \rho, \quad M_{0}>0 \Rightarrow V \leq c_{0} \frac{\exp \left(-M_{0} r\right)}{r}, \quad c_{0} \neq 0 .
\end{aligned}
$$

The above setting corresponds to the partial wave expansion for the Schrodinger equation with a central potential of a scalar particle. As usual, $\delta_{l}(k)$ is the phase shift which can be defined in terms of the Jost functions of the radial Schrodinger equation comparing the asymptotic behaviors (both at $r \rightarrow 0$ and $r \rightarrow \infty$ ) of the solution of Eq. (2) with and without potential. The angular equation gives rise, obviously, to the the spherical harmonics (and to the Legendre polynomials) which also appear in the partial-wave expansion.

The above defined partial-wave expansion satisfies the optical theorem (which is an important consequence of unitarity):

$$
\operatorname{Im} f(\theta=0)=\frac{k}{4 \pi} \sigma_{\text {Total }},
$$

where $\sigma_{\text {Total }}$ is the total cross section. In order to derive this result the only necessary ingredients are the completeness of the Legendre functions and the fact that $\delta_{l}(k)$ is real for real values of $l$ and $k$.

The important link between the radial and the angular equations (which plays a fundamental role in the Regge transform) is the (seemingly innocuous) relation in Eq. (3). In fact, as it will be shown in the next section, it is precisely this relation which is modified in the presence of monopoles (due to the non-trivial extra contributions which appear in the angular momentum operator).

One can also get a partial-wave expansion without introducing the Schrodinger equation but just 
exploiting the invariance of the $S$-matrix under spatial rotation (see, for a clear and pedagogical analysis, chapter 6 of [21]) and then expanding over the corresponding eigenvectors. The choice of the potential in Eq. (44), based on [27], is the one appearing in the pioneering papers [28] and [29]. However, the hypothesis on the potential can be somehow relaxed (see for instance [20] [21]) keeping, of course, the short-range nature of the potential.

Using standard arguments 20] 21] 22, of scattering theory from (superposition of) Yukawa's potential together with the complex angular momentum technique introduced in [28] [29] [52] one can prove that there exist a unique interpolating function which is meromorphic in $\lambda$ in the half-plane $\operatorname{Re} \lambda>0$ which reduces to the usual phase shift for integer $l$. Moreover, the analyticity (meromorphy) domain of the phase shift has been also extended to the full complex $\lambda$-plane in [53] [54] [55].

An important corollary of the results derived in [53] 54] which will be useful in the present framework is that when $\lambda$ is real $\delta(\lambda, k)$ is always real (namely, not only when $\operatorname{Re} \lambda>0$ ):

$$
\lambda \in \mathbb{R} \Rightarrow \delta(\lambda, k) \in \mathbb{R}
$$

Such a conclusion only depends on the radial Schrodinger equation (see, for instance, the first two sections of [53]).

The arguments which lead to the conclusion that, for short-range potentials, there exist a unique interpolating function $\delta(\lambda, k)$ which reduces to the physical value for integers $l$ and which is meromorphic in $\lambda$ in the full complex $\lambda$ plane depend exclusively on the radial Schrodinger equation (2). The angular part (as well as the appearance of the Legendre polynomials) only enters into the game after one plugs the phase shift into the partial-wave expansion in Eq. (11). It is in this last step that the link between the radial and angular part (expressed as a relation between $\lambda$ and $l$ which, in the usual case, is the one in Eq. (3) ) becomes very important. In particular, this implies that whenever there is scattering process in which the radial Schrodinger equation has the form in Eq. (21) with a potential of the form in Eq. (44), it is possible to define (following exactly the same arguments of [28], [29], 52], 55] and [54]) a meromorphic phase-shift $\delta(\lambda, k)$ which satisfies all the properties and the bounds described in the above mentioned references and which represents the scattering effects of the potential in Eq. (4) with respect to the free-waves (which are, of course, the solutions corresponding to $V=0)$.

Particular examples of the many results which only depend on the radial Schrodinger equation are the two very important inequalities (see [28, [29] and [52])

$$
\begin{aligned}
|[\exp (i \delta(\lambda, k))-1]| & \leq \sigma(k) \frac{\exp (-\alpha \lambda)}{\lambda^{1 / 2}}, \\
\lim _{|\operatorname{Im} \lambda| \rightarrow \infty, \operatorname{Re} \lambda \rightarrow \text { const }}[\exp (i \delta(\lambda, k))-1] & =0,
\end{aligned}
$$

where $\cosh \alpha=1+M_{0}^{2} / 2 k^{2}$. Such inequalities are very relevant both for the Regge analytic continuation of the Watson-Sommerfeld transform and for the physical interpretation of the phase-shift. An elegant proof of Eq. (8) can be found in [56]. Both inequalities are relevant to derive the Regge 
formula for the scattering amplitude.

Since Eq. (3) relates $\lambda$ (which is the variable entering in the analytic continuation of the phase shift) with $l$ (which is the discrete label which enters into the definition of the partial wave expansion in Eq. (11) through a simple linear (in particular, analytic) expression, the (analytically continued) phase shift is meromorphic in the same variable which defines the (analytic continuation of the) partial wave expansion.

In particular, all the factors (namely, $(2 l+1), P_{l}(\cos \theta)$ and $\left.f_{\lambda}(k)\right)$ which appear in each term of the partial wave expansion in Eq. (11) can be suitably extended to analytic functions of the same variable $\lambda$. Consequently, one can go (see [20] [21] [22]) from the expression in Eq. (11) into the Sommerfeld-Watson expression

$$
f(\theta, k)=\frac{1}{2 \pi k} \int_{C} \frac{\lambda d \lambda}{\cos \pi \lambda} P_{\lambda-1 / 2}(-\cos \theta)[S(\lambda, k)-1] \quad, \quad \lambda=l+\frac{1}{2},
$$

and, then, to the Regge expression (thanks to the bounds for the phase-shift found in [28, [29] and [52])

$$
f(\theta, k)=\frac{1}{2 \pi k} \int_{-\infty}^{+\infty} \frac{\lambda d \lambda}{\cos (i \pi \lambda)} P_{i \lambda-1 / 2}(-\cos \theta)[S(i \lambda, k)-1]-\frac{i}{k} \sum_{j=1}^{N} R_{j} P_{\alpha_{j}}(-\cos \theta) .
$$

In Eq. (9) the circuit $C$ rounds counterclockwise the zeros of $\cos \pi l$.

The representation in Eq. (10) is called Regge formula for the scattering amplitude. The circuit of the first term on the right hand side of Eq. (10) corresponds to the line $\operatorname{Re} \lambda=0$ (together with a semi-circle at infinity which, however, does not contribute due to the inequality (77)).

The first term on the right hand side of Eq. (10) is called background integral and it gives small contribution for large $\cos \theta$ as it decreases as $|\cos \theta|^{-1 / 2}$ (due to the fact that the Legendre polynomial within the integral in Eq. (10) has index $i \lambda-1 / 2$ with $\lambda$ real). As it has been already emphasized, in the usual case, it is possible to push further to the left the integration path of the background integral [53] 54 [55. This allows to make the contribution from the background integral even smaller (as the label $i \lambda-1 / 2$ of the Legendre polynomial would go into $i \lambda-K$ with $K>1 / 2$ ). However, as it will be shown in the next sections, the presence of monopoles represents an obstruction in pushing the background integral to the left.

The second term on the right hand side of Eq. (10) corresponds to the contributions arising from the Regge poles (all the factors-but the Legendre polynomial evaluated at the Regge poles-have been packed into $R_{j}$ ).

At last, three important asymptotic properties of the Legendre functions which are needed to prove 
the above well-known results are:

$$
\begin{aligned}
& P_{l}(\cos \theta) \underset{l \rightarrow \infty}{\sim} \frac{\sigma(E)}{l^{1 / 2}} \exp [|\operatorname{Im} \theta| l], \\
& \left|\frac{P_{l}(-\cos \theta)}{\sin \pi l}\right| \underset{\operatorname{Im} l \stackrel{\infty}{\rightarrow} \pm \infty}{\leq} \frac{b(\theta)}{l^{1 / 2}} \exp [-|\operatorname{Re} \theta \operatorname{Im} l|], \\
& P_{l}(z) \underset{|z| \rightarrow \infty}{\sim} a(l) z^{l},
\end{aligned}
$$

where the precise forms of the bounded functions $\sigma(E), b(\theta)$ and $a(l)$ are not relevant.

\section{Scattering from Monopoles and Yukawa potentials}

In the presence of a Dirac monopole the angular momentum operator is the following (see for a detailed review [3]):

$$
\vec{J}=\vec{l}-\mu \frac{\widehat{r}+\widehat{z}}{1+\cos \theta}=J_{\theta} \widehat{e}_{\theta}+J_{\varphi} \widehat{e}_{\varphi}+J_{r} \widehat{e}_{r}, \quad \mu=\frac{e g}{c \hbar}
$$

where $\vec{l}$ is the standard orbital angular momentum, $e$ and $g$ are the electric and magnetic charges, $\widehat{r}$ and $\widehat{z}$ are the unit vectors in the radial and $z$ directions respectively, $\mu$ is the quantized strength of the monopoles

$$
|\mu|=\frac{1}{2}, 1, \ldots,
$$

while the $\widehat{e}_{k}$ are the spherical unit vectors. Without loss of generality, we can assume that $\mu>0$ as the analysis with $\mu<0$ is similar. The components of the total angular momentum operator $\vec{J}$ read

$$
J_{\theta}=\frac{1}{\sin \theta}\left(i \partial_{\varphi}+\mu(1-\cos \theta)\right), \quad J_{\varphi}=-i \partial_{\theta}, \quad J_{r}=-\mu
$$

Consequently, the centrifugal barrier in the Schrodinger equation is determined by $\vec{J}^{2}$. The eigenvectors and eigenvalues of $\vec{J}$ are the so-called rotation matrices or generalized spherical harmonics (the conventions and normalizations coincide with [24] and [26]):

$$
\begin{gathered}
\vec{J}^{2} d_{\mu m}^{(l)}=s d_{\mu m}^{(l)}, \quad x=\cos \theta, \quad s=l(l+1), \quad l=n-\mu, n \in \mathbb{N}, l \geq \mu,-l \leq m \leq l, \\
d_{\mu m}^{(l)}(\theta, \varphi)=N_{\mu l m} \exp [i(\mu+m) \varphi](1-x)^{\frac{(\mu+m)}{2}}(1+x)^{\frac{(\mu-m)}{2}} P_{l-m}^{(\mu-m, \mu+m)}(x) \\
P_{n}^{(\alpha, \beta)}(x)=\frac{(-1)^{n}}{2^{n} n !}(1-x)^{-\alpha}(1+x)^{-\beta} \frac{d^{n}}{d x^{n}}\left[(1-x)^{\alpha+n}(1+x)^{\beta+n}\right], l=\mu, \mu+1, \mu+2, \ldots, \\
N_{\mu l m}=\left[\frac{(l-m) !(l+m) !}{(l-\mu) !(l+\mu) !}\right]^{-1 / 2},
\end{gathered}
$$

where the $P_{n}^{(\alpha, \beta)}$ are the Jacobi polynomials, the $d_{\mu m}^{(l)}$ are the generalized spherical harmonics or $d$-rotation matrices (obviously, when $\mu=0$ the above expressions reduce to the usual Legendre Polynomials and spherical harmonics). An important property of the Jacobi polynomials is that they are 
entire functions of the three indices $\alpha, \beta$ and $n$. The $P_{n}^{(\alpha, \beta)}$ form a complete set for any fixed $\alpha$ and $\beta$. As it has been already emphasized (the cleanest discussion is probably the one in [10]) the extra term in the above defined angular momentum operator $\vec{J}$ (which obviously is directly related to the gauge field of the Dirac monopole) is responsible for the fact that (depending on the strength of the Dirac monopole) one scalar particles in the field of a monopole can behave as Fermions. This consideration together with the fundamental role of the angular momentum in the Regge formalism [28] [29] strongly suggest that it may be a good idea to try to apply Regge theory in the presence of topological solitons. It is also interesting to note that in the cases of Skyrmions and of a 't Hooft-Polyakov monopoles, the corresponding angular momentum operators get similar extra terms related to the topological charges (see 6] and [14 [15] [16]). Moreover, far from the core of a 't Hooft-Polyakov monopole (see section 6 of [25]) the scattering problem of a scalar field charged under the $S U(2)$ gauge group in the field of the non-Abelian monopole itself reduces precisely to the scattering problem off a Dirac monopole. This observation (together with the essential question about the role of the short-range interactions related to the Higgs field in the realistic case in which the potential is non-vanishing) has been one of the main motivations of the present work.

Three crucial properties of the Jacobi polynomials (see [57] as well as the appendix of [58]) which allow the Watson-Sommerfeld-Regge transform in the monopole case (as it will be described in the next sections) are the analogue of Eqs. (11), (12) and (13) for the Legendre polynomials:

$$
\begin{aligned}
& P_{l}^{(\alpha, \beta)} \underset{l \rightarrow \infty}{\sim} \frac{\sigma_{\alpha \beta}(E)}{l^{1 / 2}} \exp [|\operatorname{Im} \theta| l], \\
& \left|\frac{P_{l}^{(\alpha, \beta)}}{\sin \pi l}\right| \underset{\operatorname{Im} l \rightarrow \pm \infty}{\leq} \frac{b_{\alpha \beta}(\theta)}{l^{1 / 2}} \exp [-|\operatorname{Re} \theta \operatorname{Im} l|], \\
& P_{l}^{(\alpha, \beta)} \underset{|z| \rightarrow \infty}{\sim} a_{\alpha \beta}(l) z^{l},
\end{aligned}
$$

where the precise forms of the bounded functions $\sigma_{\alpha \beta}(E), b_{\alpha \beta}(\theta)$ and $a_{\alpha \beta}(l)$ are not relevant. Thus, for fixed values of the quantized monopoles strength $\mu$, the Jacobi polynomials satisfy exactly the same asymptotic bounds as the usual Legendre ones. Not so surprisingly, the above asymptotic behavior of the deformed spherical harmonics are some of the key mathematical properties which allow the extension of the Regge formalism of complex angular momenta to helicity amplitudes [39] for particles with spin (see [59] 660] 61] 62]; a nice and elegant group-theoretical formulation of the Regge formalism for helicity amplitude is 63] 64 [65]). What is more interesting is that the above asymptotic properties of the generalized spherical harmonics will be also crucial for the extension of the Regge formalism to the scattering problem in the presence of both monopoles and short range potentials. This, perhaps, could have been guessed on the basis of [10]. However, a crucial difference between the usual helicity amplitudes and the scattering amplitudes in the presence of monopoles and short range potential (a sort of fingerprint of topologically non-trivial configurations) will be apparent when discussing the analytic continuation a la Regge of the scattering amplitude. 


\subsection{The Schrodinger equation with monopoles and short range potentials}

Thus, the Schrodinger equation in the electromagnetic field of a Dirac monopole $\mathbf{A}$ of the form

$$
\mathbf{A}=\frac{g}{r} \frac{1-\cos \theta}{\sin \theta} \widehat{e}_{\varphi}
$$

( $g$ being the magnetic charge) and a central potential reads

$$
-\frac{1}{2 M}\left[(\vec{\nabla})^{2}-V(r)\right] \Psi(\vec{r})=E \Psi(\vec{r}), \quad E=\frac{k^{2}}{2 M}
$$

where, according to the minimal coupling rule, one has

$$
\vec{\nabla}=\vec{\partial}-i e \vec{A}
$$

In the above formula, $\vec{\partial}$ is the flat spatial gradient and $\vec{A}$ is the gauge potential of the Dirac monopole in Eq. (23). It is worth to note that the most elegant and mathematically sound procedure to define the gauge potential of a Dirac monopole is the one based on the theory of fiber-bundle introduced by $\mathrm{Wu}$ and Yang in [66] 67]. One of the main advantages of such formulation is that it avoids the use of singular gauge potentials (such as the one in Eq. (23)). On the other hand, as far as the Schrodinger equation is concerned, the local and the Wu-Yang approaches produce the same result (as it can be seen by comparing Eq. (53), section 11 of [67] with Eqs. (25) and (27) here below).

After standard manipulations (see the original papers [8] and [9] as well as the nice review [3]), it is possible to bring Eq. (24) into the following form

$$
-\frac{1}{2 M}\left[\frac{1}{r^{2}} \partial_{r}\left(r^{2} \partial_{r}\right)-\frac{(\vec{J})^{2}-\mu^{2}}{r^{2}}-V(r)\right] \Psi(\vec{r})=E \Psi(\vec{r}), \quad E=\frac{k^{2}}{2 M}
$$

where the total angular momentum operator $\vec{J}$ is defined in Eq. (14) while the potential is a superposition of Yukawa potentials as in the original analysis of [28], 29] and [52]:

$$
V(r)=\int_{M_{0}}^{\infty} \sigma(\rho) \frac{\exp [-\rho r]}{r} d \rho, \quad M_{0}>0 \Rightarrow V \leq c_{0} \frac{\exp \left(-M_{0} r\right)}{r}, c_{0} \neq 0 .
$$

The above equation is separable and, with standard methods (see, for instance, [3]), one gets the following radial and angular equations by separation of variables

$$
\Psi(\vec{r})=\frac{\psi_{k \lambda}(r)}{r} d_{\mu m}^{(l)}(\theta, \varphi)
$$




$$
\begin{aligned}
k^{2} \psi_{k \lambda} & =\left[-\frac{d^{2}}{d r^{2}}+\frac{\lambda^{2}-\frac{1}{4}}{r^{2}}+V\right] \psi_{k \lambda}, \\
\lambda & =\lambda(l)=\sqrt{\left(l+\frac{1}{2}\right)^{2}-\mu^{2}}, \quad l \geq \mu \Rightarrow \lambda>\mu, \\
s d_{\mu m}^{(l)} & =\vec{J}^{2} d_{\mu m}^{(l)},
\end{aligned}
$$

where the generalized spherical harmonics $d_{\mu m}^{(l)}$ have been discussed in Eqs. (16), (17) and (18). It is worth emphasizing that, due to the inequalities in Eqs. (16) and (28), the centrifugal barrier in the monopole case never vanishes. This is one of the consequences of the fact that the generators of the angular momentum operator get an extra contribution from the topologically non-trivial configurations.

The Regge formalism discloses a crucial difference between the usual cases and the present case of a scattering problem off a topologically non-trivial scatterer. Such a difference is related to the functional relation which links the parameter $\lambda$ which appears in the radial Schrodinger equation with the parameter $l$ which determines both the eigenvalues of the angular part and the discrete label of the partial-wave series. As it has been already emphasized, in the usual case such link between the radial and angular part (namely, Eq. (3) ) is expressed by an analytic relation between $\lambda$ and $l$. In the present case, the link between the radial and angular equations is instead represented by Eq. (28) which is not anymore an analytic relation between $\lambda$ and $l$. The origin of such a "mismatch" between $\lambda$ and $l$ is the fact that the topological defect is spatially spherically symmetric only up to internal transformation and, consequently, the centrifugal barrier is modified in such a way to generate a fixed cut in the complex angular momentum plane. This simple observation allows to determine a peculiar fingerprint of a scattering process off a topologically non-trivial object as it will be discussed in the next subsections.

\subsection{Partial-wave expansion for scattering off monopoles and short range potentials}

Here, the analysis of chapter 6 of [21] will be followed taking into account that, in the presence of a topological soliton such as a monopole, the angular momentum gets an extra contribution from internal symmetries. In the present case, the scattering matrix $S$ is invariant under the following rotation operator $R(\alpha)$ :

$$
\begin{aligned}
R & =R(\alpha)=\exp (-i \alpha \vec{J}), \\
R^{\dagger} S R & =S,
\end{aligned}
$$

where $J$ is the total angular momentum defined in Eq. (14) (which receives contributions from the monopole as well). The next step in order to define the partial-wave expansion is to define the free waves or asymptotic states. The best choice is, obviously, to define as free waves the eigenfunctions 
of $H_{0}, J^{2}$ and $J_{z}$. However, it is worth emphasizing that this choice implies that one has to choose as free Hamiltonian $H_{0}$ the Hamiltonian without the potential (namely, $V=0$ in Eqs. (25) and (27)) but with the effects of the monopole (which are encoded in the modified centrifugal barrier and in the replacement of the Legendre polynomials with the Jacobi polynomials) included.

The reason why this choice is very convenient 4 is that if one would include the effects of the monopole into the interaction, then the angular symmetry generators of the free Hamiltonian (which, in such a case, would be just the free Laplacian) would be different from the angular symmetry generators of the full Hamiltonian. A more concrete way to see this is to notice that the effects of the monopole are not small as they are described by a parameter ( $\mu$ in our case) which is quantized, thus it cannot be reduced continuously to zero. Even from the perspective of the Bethe-Salpeter equation with singular potentials (see [72] [73]), the present choice is mandatory. Namely, in order to construct well-defined solutions for the Bethe-Salpeter equation with potentials which at the origin behave as $1 / r^{2}$ (which is basically the present case; on the other hand the authors of that references also dealt with more singular potential at the origin), one must include the singular potential into the free Hamiltonian (see in particular section 3 of [72]). Thus [72] [73] tell that, in the case of a monopole and a short range potential, the free Hamiltonian is the one without the short range potential but with the effects of the monopole included 5 .

Moreover, the standard procedure (for a pedagogical review see [71]) to compute any observable in the path integral formalism is to consider the perturbative expansion within the given topological sector. This means, in particular, that the definition of free wave functions depend on the topological sector which one is considering. There is an undeniable physical reason for this already in the case of a Dirac monopole. One can feel the effects of the monopole even from arbitrary far (for a pedagogical review see the first two chapters of [3]) both classically (as a classical particle in the field of a monopole is constrained to move on a cone) and quantum mechanically (for instance, there is no $s$-wave in the field of the monopole as the centrifugal barrier never vanishes). This is why the definition of "free wave function" should depend on the topological sector one is considering.

Therefore, in the cases in which there is both a monopole and a short range potential, it only makes sense to consider as "free waves" the wave functions in the presence of the monopole but without the potential. In other words, in the present case, the free waves correspond to the scattering monopole wave functions constructed in the foundational references [8] [9] [23] [24] [25].

\footnotetext{
${ }^{4}$ This is actually mandatory if one wants to apply Regge theory when there is both a topological soliton and a short range potential: the effects of topological solitons are not short-range as one needs in Regge theory. However, as they can be encoded into the angular momentum, such effects can be included in the free hamiltonian. Thus, the interaction term is now short range and the Regge theory can be applied.

${ }^{5}$ It is worth to emphasize that to take as free Hamiltonian the one with the effects of the monopole included is a mathematically well-defined procedure as the chosen free Hamiltonian has not bound states. In the cases in which bound states are present, some extra care is needed.
} 
For the above reasons, the spherical basis which will be used to diagonalize the $S$ matrix will be

$$
\begin{aligned}
\langle\vec{x} \mid E, l, m\rangle & =i^{j}\left(\frac{2 m}{\pi p}\right)^{1 / 2} \frac{\widehat{j}_{\lambda}(k r)}{r} d_{\mu m}^{(l)}, \\
\left\langle E^{6}, l^{\natural}, m^{6} \mid E, l, m\right\rangle & =\delta\left(E^{6}-E\right) \delta_{l l^{\natural}} \delta_{m m^{6}}
\end{aligned}
$$

where $\widehat{j}_{\lambda}(k r)$ are the Riccati-Bessel function while the $d_{\mu m}^{(l)}$ are the rotation matrices in Eq. (29). Hence, the phase shift and the scattering amplitude constructed in the present paper describe the effects of the short range potential on top of the monopole scattering wave functions of [8] [9] [23] [24] [25]. In this basis, the $S$-matrix is diagonal as it commutes with $H_{0}$ and $\vec{J}$ :

$$
\begin{aligned}
\left\langle E^{\natural}, l^{\natural}, m^{\natural}|S| E, l, m\right\rangle & =\delta\left(E^{\natural}-E\right) \delta_{l l^{\natural}} \delta_{m m^{\natural}} S_{l}(E) \\
S_{l}(E) & =\exp [2 i \delta(l, E)]
\end{aligned}
$$

where in Eq. (33) it has been explicitly taken into account the unitarity of the $S$-matrix. From this definition, one can immediately derive a partial wave expansion expressing the scattering amplitude in the momentum basis (which is directly related to the observable cross section: see [21] for a pedagogical exposition). The only technical detail which must be taken into account is that, as for the usual spherical harmonics, also the deformed spherical harmonics form a complete set. Thus, the partial wave reads in this case

$$
f_{\mu}(k, x)=\frac{\exp (-i \pi \mu)}{k}\left(\frac{1-x}{2}\right)^{\mu} \sum_{l=0}[2(l+\mu)+1] \exp [i \delta(l, k)] \sin \delta(l, k) P_{l}^{2 \mu, 0}(x) .
$$

The optical theorem (which discloses one of the most relevant differences between the usual case and the Regge theory in presence of topological solitons) will be discussed in the next sections separately for the $\mu=1 / 2$ case and the higher values of $\mu$.

In the "Reggeization" of helicity amplitudes (see in particular [60] [61] [62]) it is customary to define the scattering amplitude without the "kinematical factors" $\left(\frac{1-x}{2}\right)^{\mu}$ of the rotation matrices which may lead to extra singularities in the scattering amplitude like branch cuts. On the other hand, if one only keeps the Jacobi polynomial in the definition of the scattering amplitude then only dynamical singularities appear. In the following only dynamical singularities will be discussed.

\subsection{Phase shift and Schrodinger equation}

The only missing piece of information that one needs to discuss the scattering amplitude using the Regge transform is the phase shift defining the $S$-matrix elements which enters in the scattering amplitude in Eqs. (33) and (34).

The radial Schrodinger equation (27) is the same as in the usual case in Eq. (22). Thus, using the same arguments of [52] (based on the analysis of the Jost functions associated to Eq. (27)) one can define a unique interpolating phase shift $\delta(\lambda, k)$ which is meromorphic in the full complex $\lambda$-plane (following the same arguments in [53] [54]) and which fulfills all the bounds derived in [28] [29] [52]. 
One can also prove that the number of Regge poles is finite (in the case of Yukawa's potential) and that the inequalities in Eqs. (77) and (8) are satisfied. Consequently (taking into account the well-known properties of the Jacobi polynomials in Eqs. (20), (21) and (22) which are completely analogous to the properties of the usual Legendre polynomials needed for the Regge transform) all the mathematical ingredients necessary in order to perform the Watson-Sommerfeld-Regge transform of the scattering amplitude in Eq. 34) are available.

However, the big difference with respect to the usual case is that the variable $\lambda$ is related to the label $l$ of the angular functions by a non-analytic relation: Eq. (28). Hence, the phase-shift $\delta(l, k)=\delta(\lambda(l), k)$ as function of the angular label $l$ is meromorphic in the complex $l$-plane with a branch cut on the real $l$ axis from $-|\mu|-1 / 2$ to $|\mu|-1 / 2$. Therefore, unlike what happens in [53] [54, it will not be possible to push further to the left the integration path of the background integral in the Regge formula with monopoles.

It is also worth emphasizing the difference of the present situation with respect to previous analysis of the Regge formalism with singular potentials at the origin (see, in particular, [74] [75] [76] [77] [78]) in which the usual Regge formalism (namely, without any topological soliton and keeping both the standard generators of the angular momentum and the Legendre polynomial in the partial wave expansion) was extended to include potentials of the form

$$
V_{S}=\frac{A}{r^{2}}
$$

The physical results and their interpretations within that references (see, in particular [74] [75] [76]) are basically opposite to the present results (which strongly depend on the role of the monopole and of the corresponding modified angular momentum generator and Jacobi polynomials). In particular, in the usual case with singular potential considered in [74] [75] [76] one has to interpret the singular potential as attractive when $A>0$ and as repulsive when $A<0$. Consequently, in that references, the fixed cut in the angular momentum plane 6 which is generated by the singular potential is interpreted as a sign of the attractive nature of the singular potential itself. A further consequence of the results in that references is that one should not consider the case $A>1 / 4$ as "the system collapse into the center and the very concept of scattering is no longer clear" (this quotation is from [76] but similar results are contained in [74] [75] [78]).

On the other hand, the present situation is practically the opposite. At a first glance, if one look at the Schrodinger equation in the presence of both a monopole and a short range potential in Eq. (25) one could naively think that the effect of the monopole is to decrease the centrifugal barrier due to the extra potential

$$
V_{M}=\frac{\mu^{2}}{r^{2}}
$$

which has always the opposite sign with respect to the usual centrifugal barrier. In fact, as it is

\footnotetext{
${ }^{6}$ In Regge theory applied to QFT, the appearance of cuts in the complex $\lambda$ plane is a well known phenomenon (see, in particular, the discussion in 68] 69, [70]). The usual situation is that such branch points are moving (in the sense that they depend on the Mandelstam variables). On the other hand, the cuts considered here are fixed due to their topological origin.
} 
well known, this interpretation is completely wrong since the effect of the monopole (as well as of all the relevant topological solitons in $3+1$ dimensions) is actually to increase the centrifugal barrier. The information about the increase of the centrifugal barrier lies in the link between the radial and angular equations. Unlike the setting considered in [74] [75] [76] 78, the effects of the monopole do not reduce just to the extra term in Eq. (36). The angular equation and the corresponding eigenvalues are affected by the presence of the monopole as well. This fact has important consequences: first of all, in the present case there are not extra bound states so that the scattering problem is well defined for any physical value of $\mu$. Secondly, as far as the cut is concerned, due the fact that $\mu \geq 1 / 2$ we are precisely in the range which was not considered in [74] [75] [76] [78] (namely, $A>1 / 4$ in the notation of [76]) and yet, of course, there will be no "collapse of the wave function on the center" (as guaranteed by the angular part of the monopole-short range potential problem).

The physical consequences of these facts will be discussed in the next section.

\section{Monopole Regge formula and the fixed cut}

In the previous section it has been shown that one can define the phase shift for a scattering problem off a monopole and a short range potential and that the phase shift satisfies, in the natural variable $\lambda$, all the bounds derived in the classic papers [28] [29] [52] [53] [54]. Such results together with the well known bounds on the Jacobi polynomial (see Eqs. (20), (21) and (22): useful references are [57] as well as the appendix of [58]) do allow a Watson-Sommerfeld-Regge transform. However, in the present case, there is an extra ingredient (which is a characteristic fingerprint of the presence of topological solitons in a scattering problem) which is absent in the usual helicity amplitudes analyzed in [59] 60] 61] 62].

Due to the fact that the relation between $\lambda$ and $l$ has the form in Eq. (28), in the monopole case there is a fixed cut in the complex $l$ plane along the real $l$ axis from $-|\mu|-1 / 2$ to $|\mu|-1 / 2$. Thus, such a fixed cut opens up due to the presence of a monopole and its size is $2|\mu|$. Consequently, as $2|\mu|$ is at least 1 , the cut will touch at least one of the poles of the factor $1 / \cos \pi \lambda$ which appears in the Sommerfeld-Watson transform (see Eq. (9)). This fact prevents one from pushing the integration path of the background integral in the Regge transform further to the left. Thus, the procedure of [62] (which is common for helicity amplitudes) cannot be used in this case. In a sense, the presence of monopoles can be seen as an obstruction to the maximal analyticity principle in the complex $l$ plane advocated by Chew and Frautschi [79] [80].

It is worth emphasizing that the results of [26] (in which the authors only analyzed the scattering off a monopole without any short-range interaction and, consequently, without using the Regge approach) are closely related to the present ones. The reason is that the origin of the branch cut singularities of the scattering amplitude in $\cos \theta$ found in [26] is the same as the origin of the fixed branch cut in the complex $l$ plane found in the present work: both of them are related to the mismatch in Eq. (28) between $\lambda$ and $l$ in the presence of a non-trivial topological structure. It seems that the Regge theory is a more suitable tool to disclose the physical implication of such a result (as, for instance, it sheds considerable light on the asymptotic behavior of the scattering amplitude). 


\subsection{The case $\mu=1 / 2$}

Let us first consider the case in which $\mu=1 / 2$ (which is the lowest possible non-trivial value of the monopole strength). Besides the intrinsic interest of this case, from the point of view of non-Abelian theories, it describes the asymptotic behavior of a scattering off a 't Hooft-Polyakov monopole of unit charge (namely, the stable non-Abelian magnetic monopole) far from its core [25].

The first consequence of the presence of the fixed cut along the real $l$ axis is that, in order to perform the Sommerfeld-Watson transform the analogue of the circuit $C$ in Eq. (9) (which in the usual case rounds all the integers including 0) now must exclude 0 (namely, the first term in the partial wave expansion in Eq. (34)) as the cut begins at $l=-1$ and ends at $l=0$.

Nevertheless, it is important to emphasize (as one can check directly) that due to both the unitarity of the $S$-matrix elements (namely $\delta(\lambda, k)$ is real for $k$ and $\lambda$ real 7 ; in particular, for $k$ real, $\delta(\lambda(l), k)$ is real for $l=0,1,2, \ldots$ so that in each term of the partial wave expansion in Eq. (34) the phase shift is real, see Eq. (6) ) and to the completeness relations satisfied by the Jacobi polynomial the above scattering amplitude satisfies the optical theorem:

$$
\operatorname{Im} f(k, \theta=0)=\frac{k}{4 \pi} \sigma_{\text {Total }} .
$$

Thus, the Sommerfeld-Watson transform of Eq. (34) (in this section the extra kinematical factors of the rotation matrices will not be considered) reads

$$
\begin{aligned}
&\left.f_{\mu}(k, x)\right|_{\mu=1 / 2}=2 \exp [i \delta(0, k)] \sin \delta(0, k) P_{0}^{1,0}(x)+ \\
& \int_{C_{1 / 2}} \frac{[2(l+\mu)+1] d l}{\sin \pi l} P_{l}^{1,0}(-\cos \theta)[S(\lambda(l), k)-1],
\end{aligned}
$$

where it has been taken into account that $\lambda\left(l=0, \mu=\frac{1}{2}\right)=0$ and it has been explicitly emphasized that the phase shift depends on $l$ through $\lambda$. Thus, the first term of the partial wave expansion cannot be encoded in the Sommerfeld-Watson integral. On the other hand, such a term is well behaved as the phase shift $\delta(0, k)$ is well behaved (and approaches to 0) for large $k$ (see, for instance, 20]).

The Sommerfeld-Watson integral in Eq. (37) can be written in the Regge form ([28] [29] [52]):

$$
\begin{gathered}
\left.f(k, x)\right|_{\mu=1 / 2}=2 \exp [i \delta(0, k)] \sin \delta(0, k) P_{0}^{1,0}(x)+ \\
+\frac{1}{2 \pi k} \int_{C_{\varepsilon}} \frac{[2(l+\mu)+1] d l}{\sin \pi l} P_{l}^{1,0}(-x)[S(\lambda(l), k)-1]-\frac{i}{k} \sum_{j=1}^{N} R_{j} P_{\alpha_{j}}^{2 \mu, 0}(-x), \varepsilon \in \mathbb{R}_{+} .
\end{gathered}
$$

In the above equations, the analogue of the $\operatorname{Re} \lambda=0$ line for the background integral in the usual case

\footnotetext{
${ }^{7}$ It is useful to remind that the validity of such a result only depends on the radial Schrodinger equation (see, for instance, the first two sections of [53]). Consequently, it also holds in the present case.
} 
(see Eq. (10)) is the line $C_{\varepsilon}$ defined by the equation

$$
\operatorname{Re} l=\varepsilon,
$$

where $\varepsilon$ is a positive arbitrarily small but non-vanishing number (one cannot take $\varepsilon=0$ otherwise one would touch the cut which begins at $l=0$ ). Moreover, it is easy to check that when $\operatorname{Im} l \rightarrow \pm \infty$ with $\operatorname{Re} l=\varepsilon$, one gets

$$
\lim (\operatorname{Im} \lambda) \rightarrow \pm \infty, \lim (\operatorname{Re} \lambda) \rightarrow \frac{1}{2},
$$

so that the well known bounds (see [56] [20]) for $\delta(\lambda, k)$ when $|\operatorname{Im} \lambda| \rightarrow \infty$ with $\operatorname{Re} \lambda$ fixed (which ensure the convergence of the background integral) can be applied

Thus, the remarkable effect is that, unlike what happens in the usual cases [28] 29], the background integral does not decrease anymore for large values of $|\cos \theta|$. The obvious reason is that the Jacobi polynomial in the background integral in Eq. (38) is $P_{\varepsilon+i y}^{1,0}(-x)$ (with $\varepsilon$ arbitrarily small, real and positive and $y$ real varying from $-\infty$ to $+\infty$ ). Hence, one does not get the usual decreasing behavior for large values of $|\cos \theta|$. Rather, an oscillating non-decreasing behavior for large values of $|\cos \theta|$ appears. Thus, at low transferred momentum the behavior of the scattering amplitude is not dominated by the leading Regge trajectory and the background integral also plays a key role (compared with the standard case). It is only when the leading Regge trajectory $\alpha_{P}(t)$ (which, in a relativistic context, would correspond to the Pomeron) becomes positive enough that the Regge pole behavior dominates the background integral.

\subsection{Monopoles of higher charges}

Here the case of higher values of $\mu$ are analyzed. From the point of view of non-Abelian theories, this would correspond to (the large $r$ behavior of) non-Abelian monopoles which are spherically symmetric and, at the same time, have higher topological charges. In fact, such spherically symmetric hedgehog configurations do not exist8. An easy way to argue that this is the case is to observe that such configurations would be highly unstable (the same is true even if one gives up spherical symmetry requiring just axial symmetry: see [3] [4]).

As from the point of view of non-Abelian theories the case of $\mu>1 / 2$ is completely different from the case $\mu=1 / 2$, it is natural to wonder whether the present approach is able to detect in a direct way such a difference. Interestingly enough, within the present approach, the difference between these two cases emerges in a very natural and intuitive way. When $\mu=1,3 / 2, \ldots$ it is trivial to see that the first terms (corresponding to the lowest $l$ ) in the partial wave expansion in Eq. (34) would have a complex $\lambda(l)$ and, consequently, a complex phase shift $\delta(\lambda(l), k)$ which signals instabilities (see the nice discussion in [81]). In particular, the optical theorem does not hold in such cases. Thus, one can derive a well defined partial wave expansion and Regge continuation to complex angular momenta only in the presence of stable topological defects.

\footnotetext{
${ }^{8}$ Stable multi-monopoles solutions with higher topological charges do indeed exist. However, such solutions are not spherically symmetric (see [3]). Consequently, when stable non-Abelian multi-monopole configurations are involved, the partial wave expansion should be modified in order to take into account the lack of spherical symmetry.
} 


\section{On the range of validity of the approach}

As discussed in details in [25], the scattering from a Dirac monopole provides with a accurate description of the scattering form non-Abelian monopole far from the core of the latter. In terms of energy bounds, this means that the present results can be a valid description of scattering process in non-Abelian theories when

$$
s<M_{m o n}
$$

where $s$ is the center-of-mass Mandelstam variable and $M_{m o n}$ is the mass scale which characterizes the non-trivial topological structure of the non-Abelian theory.

The second limitation on the range of applicability of the present results in non-Abelian theories arises from the requirement that loops effects should be small. A necessary condition in order for this to happen in two-body scattering is that

$$
\left|\frac{t}{s}\right| \ll 1
$$

where $t$ is the transferred momentum Mandelstam variable. It is worth to emphasize that the above condition is nothing but the definition of the Regge region.

It is interesting to note that one of the most interesting and still open issues in the application of Regge theory in QCD lies well within the range defined by Eqs. (40) and (41). Such an issue has to do with the soft Pomeron [40] (detailed reviews are 41] 42]). Consequently, the present results on the effects of topological solitons in Regge theory (which can be applied in QFT only when the condition (40) holds and $t$ is small enough to make negligible all loops corrections) can be relevant from the soft Pomeron perspective.

It is by now quite clear that the actual behavior of the scattering amplitude at $t=0$ is much more complicated than the one in [82] (see, for instance, [83] [84]). Even if it is well-known (see [44] and references therein) that, at the scale of the soft Pomeron, the total cross section is actually very far from saturating the unitarity bound [36] [43] there is still a puzzle related to the fact that while other trajectories lead to falling cross sections, the Pomeron can lead to rising cross sections. In particular, it is difficult to justify this kind of behavior in terms of a simple Regge pole. Moreover, the BFKL equation [45] [46] [47] is able to describe very well the "hard Pomeron" (namely, the Pomeron in the region in which the Mandelstam variables $s$ and $t$ are large enough to allow to neglect nonperturbative effects. However, even this approach (which should be considered as the first principle approach derived from QCD) fails when $s$ and $t$ are low enough (but still within the Regge region $|s / t| \gg 1)$.

In the presence of a monopole, the background integral in the Regge formula 9 in Eq. (38) will not decrease with $s$. Therefore, a clear-cut Regge pole behavior can only emerge when the leading trajectory begins to dominate the background integral in Eq. (38). Consequently, the present results strongly suggest a proposal which will be discussed in the next section.

\footnotetext{
${ }^{9} \mathrm{As}$ it is explained in the next section, this result together with the cut induced by the monopole on the real axis of the complex $l$-plane hold in the relativistic case as well.
} 


\section{Relativistic generalizations}

It is natural to wonder whether the present results are just a curiosity of the quantum mechanical setting considered in this paper or if they resist in a QFT context as well. One can argue as follows that the second possibility is likely to happen. When one considers the Froissart-Gribov extension of the Regge formula in the QFT scattering of scalar particles in the presence of a monopole (or any other relevant topological defect such as instantons, dyons and so on) very similar changes appear due to the fact that the monopole modifies the generators of the angular momentum operator in the QFT case too (see, for instance, [10] [25]). Indeed, according to the Gribov-Froissart procedure [30] 31] in the scalar case when the presence of a monopole is taken into account, it is natural to guess the following expression (following chapter 1 of [48]):

$$
\begin{aligned}
&\left.A_{\mu}(s, t)\right|_{\mu=1 / 2}=2 P_{l=0}^{1,0}\left(1+2 \frac{s}{t}\right) a(\lambda(0), t)+ \\
& \frac{1}{4 i} \int_{C} \frac{[2(l+\mu)+1] d l}{\sin \pi l} \sum_{\eta= \pm 1}(\eta+\exp (-i \pi l)) P^{1,0}\left(l, 1+2 \frac{s}{t}\right) a^{\eta}(\lambda(l), t)
\end{aligned}
$$

where $P^{1,0}(l, x)$ is the analytical continuation in the complex $l$-plane of the Jacobi polynomial $P_{l}^{1,0}(x)$ of indices $(1,0)$ and angular label $l$ while $a^{ \pm 1}(\lambda(l), t)$ are the analytic continuation of the even and

odd partial waves amplitudes and $\eta$ is the signature10. As in the non-relativistic case, the first term cannot be included into the Sommerfeld-Watson contour $C$ due to the cut related to the fact that the partial scattering amplitude $a(\lambda(l), t)$ depends on $l$ through $\lambda(l)$ in Eq. (28). Thus, the Regge formula in this case reads

$$
\begin{aligned}
\left.A_{\mu}(s, t)\right|_{\mu=1 / 2} & =2 P_{l=0}^{1,0}\left(1+2 \frac{s}{t}\right) a(\lambda(0), t)+\sum_{\eta= \pm 1} \sum_{n_{\eta}} c_{n_{\eta}} P^{1,0}\left(\alpha_{n_{\eta}}(t), 1+2 \frac{s}{t}\right) \\
+ & \frac{1}{4 i} \int_{\varepsilon-i \infty}^{\varepsilon+i \infty} \frac{[2(l+\mu)+1] d l}{\sin \pi l} \sum_{\eta= \pm 1}(\eta+\exp (-i \pi l)) P^{1,0}\left(l, 1+2 \frac{s}{t}\right) a^{\eta}(\lambda(l), t),
\end{aligned}
$$

where $\alpha_{n_{\eta}}(t)$ is the position of the $n_{\eta}$-th Regge pole of parity $\eta$ and all the coefficients multiplying the Jacobi polynomials evaluated at the Regge poles (compare, for instance, with Eq. (1.15) of [48]) have been denoted as $c_{n_{\eta}}$ (which depend on $\eta$ and on the transferred momentum $t$ ). Also in the relativistic case the background integral in the Regge formula is very similar to the one in Eq. (38). Since the real part of $l$ in the last term (namely, the background integral) of in Eq. (43) is positive and arbitrarily small while the imaginary part can vary from - to $+\infty$, it does not decrease when $s \rightarrow+\infty$ and a clear-cut Regge pole behavior only emerges for large enough $t$ (assuming rising trajectories).

As from the theoretical point of view the main challenge is to explain the observed behavior of the Pomeron for very low/vanishing $t$ and not too high $s$ (namely, $s$ of the order of GeV or less), the concrete proposal arising from the present results (and, in particular, Eq. (43)) is that instead

\footnotetext{
${ }^{10}$ It is worth to note that, thanks to Eq. (39), the analysis of the behavior of the partial wave amplitudes along the immaginary $l$-axis when $|l| \rightarrow \infty$ is the same as in the usual case (see chapter 1 of [48]).
} 
of parametrizing the soft Pomeron in terms of a single Regge pole (as it is done in the majority of the phenomenological papers on this topic), one should use a parametrization of the form in Eq. (43) (keeping only the leading Regge pole and the background integrals) to try to fit the proton-proton scattering data in [83]. The only missing piece of information 11 is the (re)construction of (a good phenomenological expression for) the partial scattering amplitude $a(\lambda(l), t)$ for vanishing $t$. Once this is achieved, it could be possible to reconcile the observed non-decreasing scattering amplitude at $t=0$ (thanks to the non-decreasing background integral) with a Pomeron trajectory with intercept less than 1 in agreement with unitarity (as, at least in the Schrodinger case, the optical theorem with a monopole holds). This very interesting topic is actually under investigation. It is worth to emphasize that any clear deviation from the single-pole behavior at $t=0$ would make this proposal more attractive.

From a fundamental QCD perspective, the BFKL equation (introduced in [45] [46] [47]) is the best attempt to describe the Pomeron from first principle (detailed review are [48 [49]). It describes the (Reggeized version of) the exchange of two gluons contracted in such a way to have the vacuum quantum number as the observed Pomeron. One of the basic building block of the BFKL equation is the gluon propagator. Since, in the usual gluon propagator adopted in the BFKL formalism, the internal and space-time indices are not linked in hedgehog-like style, it is impossible to incorporate into the BFKL formalism this phenomenon of spherical symmetry up to internal rotations (which is one of the characteristic fingerprints of topologically non-trivial solitons).

The most obvious way to overcome this problem is to use in the BFKL equation the gluon propagator obtained acting on the usual propagator with the singular gauge transformation 12 introduced in [85] in order to make the non-Abelian theory as "Abelian as possible" (the regularized version of this gauge choice is now known as maximal Abelian gauge). Such propagator (which would have the necessary hedgehog-like structure) would include into the BFKL analysis relevant topological informations. Another possibility would be to use in the BFKL equation the gluon propagator in the background of an instanton or a non-Abelian monopole: the gluon propagator in these cases would have the required hedgehog structure.

I hope to come back on this interesting issues in a future publications.

\section{Discussion and future developments}

In this paper the quantum mechanical scattering from a monopole has been discussed using the Regge theory of complex angular momenta. In order to apply the Regge theory in the presence of monopoles, a short range potential (chosen as a superposition of Yukawa potentials) has to be included. Such an inclusion is very welcome as it describes the typical effects of strong interactions. Moreover, it is also useful as a description of the far field behavior of the Higgs field of a 't Hooft-Polyakov

\footnotetext{
${ }^{11}$ It seems that there are not enough available data to fully reconstruct $a(\lambda(l), 0)$ yet. However, the task to build a good phenomenological expression for $a(\lambda(l), 0)$ does not appear to be out of reach.

${ }^{12}$ The price to pay of course is that such gauge transformations are singular and the corresponding singularities describe magnetic monopoles degrees of freedom.
} 
monopole.

The results of the analysis is that the scattering amplitudes in the presence of both a monopole and a short range potential are very similar to Jacob-Wick helicity amplitude. From the intuitive point of view, this formal result is quite satisfactory due to the well known fact [10] that scalars within a monopole field can behave as Fermions. However, the application of Regge analytic continuation in the complex angular momentum plane discloses a crucial difference between this case and the usual Reggeization of helicity amplitude. Namely, a fixed branch cut on the real $l$ axis of width $2|\mu|$ (where

$\mu$ is the strength of the monopole) opens up. Such a cut is related to the modified generators of the angular momentum operator in the presence of a topological soliton (and a monopole in particular). The most relevant consequence of this fact is that the background integral in the Regge formula cannot be pushed to the left. Therefore, unlike what happens in the usual case, the background integral in the Regge formula does not decrease anymore for large values of $|\cos \theta|$. Hence, a clear-cut Regge pole behavior only emerges when the transferred momentum is large enough so that the leading pole dominates on the background integral. Consequently, at low transferred momentum, the background integral plays a key role.

These results open the possibility to reconcile the observed non-decreasing scattering amplitude at $t=0$ (thanks to the non-decreasing background integral) with a Pomeron trajectory satisfying the unitarity constraint since, at least in the Schrodinger case, the optical theorem with a monopole holds. This very relevant topic is worth to be further investigated.

A further interesting issue is the analysis of the BFKL equation in the presence of non-trivial topological structures such as monopoles. The present results strongly suggest that the physical consequences of such topological solitons are especially relevant at low transferred momentum, well within the range of energies characterizing the soft Pomeron. A concrete way to include such effects into the BFKL equation has been suggested.

At last, as it has been already remarked in the introduction, topological defects in $2+1$ dimensions give rise to similar effects as far as the centrifugal barrier is concerned (a typical case being the interaction of a charge with the (2+1)-dimensional magnetic monopole described by a Chern-Simons term: see, for instance, the detailed review [50]). Correspondingly, it is a very interesting and (to the best of author's knowledge) open question to extend the Regge formalism of complex angular momenta to scattering problems by defects and short-range potential in $(2+1)$ dimensions. In particular, it would be very interesting to apply the Regge formalism to the framework of [51] in the presence of short range potentials.

\section{Acknowledgments}

The author wish to thank David Dudal, Vincent Mathieu, Pablo Pais, Luigi Rosa, Cedric Troessaert and the anonymous referee for very useful comments which improved this manuscript. This work has been funded by the Fondecyt Grants No. 1160137. The Centro de Estudios Cientıficos (CECs) is funded by the Chilean Government through the Centers of Excellence Base Financing Program of Conicyt. 


\section{References}

[1] N. Manton and P. Sutcliffe, Topological Solitons, (Cambridge University Press, Cambridge, 2007).

[2] A. P. Balachandran, G. Marmo, B. S. Skagerstam, A. Stern, Classical Topology And Quantum States, World Scientific, 1991.

[3] Y. Shnir, Magnetic Monopoles, Springer, 2005.

[4] P. Rossi, Phys. Reports 86, (1982) 317.

[5] J. Greensite, An Introduction to the Confinement Problem, Lecture Notes in Physics, Springer, 2011.

[6] T. H. R. Skyrme, Proc. R. Soc. London A 260, 127 (1961); Proc. R. Soc. London A 262 (1961) 237; Nucl. Phys. 31, 556 (1962).

[7] P.A.M. Dirac, Proc. Roy. Soc. A133 (1931) 60.

[8] P. Banderet, Helv. Phys. Acta 19 (1946) 503.

[9] I. E. Tamm, Z. Phys. 71 (1931) 141.

[10] A. Goldhaber, Phys. Rev. 140 (1965) 1407.

[11] C. Bunster, M. Henneaux, PNAS (2007) 104 (30), 12243-12249.

[12] G. 't Hooft, Nucl. Phys. B 79 (1974) 276.

[13] A.M.Polyakov, Pis'ma JETP 20 (1974) 430; Phys. Lett. B 59 (1975) 82.

[14] R. Jackiw, C. Rebbi, Phys. Rev. Lett. 36, 1116 (1976).

[15] P. Hasenfratz and G. 't Hooft, Phys. Rev. Lett. 36, 1119 (1976).

[16] A. S. Goldhaber, Phys. Rev. Lett. 36, 1122 (1976).

[17] F. Canfora, F. Correa, A. Giacomini, J. Oliva, Phys. Lett. B 722, (2013), 364.

[18] E.B. Bogomol'nyi, Sov. J. Nucl. Phys. 24 (1976) 449.

[19] M.K. Prasad and C.M. Sommerfield, Phys. Rev. Lett. 35 (1975) 760.

[20] V. De Alfaro, T. Regge, Potential Scattering, North-Holland Publishing Company, 1965.

[21] J. R. Taylor, Scattering Theory, Dover Publications (2000).

[22] R. G. Newton, Scattering Theory of Particles and Waves, Dover Publications (2002).

[23] K. Ford, J. Wheeler, Annals of Physics 7, (1959), 259-286; ibid. 7, (1959), 287-322. 
[24] J. Schwinger, K. Milton, W. Tsai, L. DeRaad, D. Clark, Annals of Physics 101 (1976) 451.

[25] D.G. Boulware, L. S. Brown, R. N. Cahn, S. D. Ellis, C. Lee, Phys. Rev. D 14 (1976) 2708.

[26] A. P. Balachandran, S. Borchardt, S. S. Chang, A. Stern, R. Cahalan, R. Ramachandran, H. Rupertsberger, Phys. Rev. D 17 (1978) 1150.

[27] J. Charap, S. Fubini, Il Nuovo Cimento 14 (1959), 540; ibid. 15 (1960), 73.

[28] T. Regge, Il Nuovo Cimento 14 (1959), 951.

[29] T. Regge, Il Nuovo Cimento 18 (1960), 947.

[30] V. N. Gribov, Zh. Eksp. Teor. Fiz. 41, 667 (1961) [transl. Sov. Phys. JETP 14, 478 (1962)].

[31] M.Froissart, Report to the La Jolla Conference on the Theory of Weak and Strong Interactions, La Jolla,1961.

[32] M. Gell-Mann, M. L. Goldberger, F. E. Low, E. Marx, F. Zacariasen, Phys. Rev. 133, B145 (1964).

[33] G. F. Chew, S. C. Frautschi, Phys. Rev. Lett. 7, 394 (1961).

[34] G. F. Chew, S. C. Frautschi, S. Mandelstam, Phys. Rev. 126, 1202 (1962).

[35] S. Mandelstam, Phys. Rev. 112, 1344 (1958); ibid. 115, 1741 (1959); ibid. 115, 1752 (1959).

[36] M. Froissart, Phys. Rev. 123 (1961) 1053.

[37] V. N. Gribov, The Theory of Complex Angular Momenta: Gribov Lectures on Theoretical Physics, Cambridge University Press (2003).

[38] W. Drechsler, Fort. Der Physik 18, (1970), 305-448.

[39] M. Jacob, G. Wick, Ann. of Phys. 7, 404 (1959).

[40] I.Y. Pomeranchuk, Sov. Phys. 3, 306 (1956); Sov. Phys. 7, 499 (1958).

[41] Otto Nachtmann, "Pomeron Physics and QCD" arXiv:hep-ph/0312279.

[42] A. Donnachie, H. G. Dosch, P. V. Landshoff, O. Nachtmann, Pomeron Physics and QCD, Cambridge University Press, 2002.

[43] A. Martin, Phys. Rev. 129, (1963) 1432.

[44] A. Dymarsky, JHEP 07 (2015) 106.

[45] I I Balitsky and L N Lipatov, Sov. J. Nucl. Phys. 28, 822 (1978).

[46] E A Kuraev, L N Lipatov and V S Fadin, Sov. Phys. JETP 45, 199 (1977). 
[47] V S Fadin and L N Lipatov, Phys. Lett. B 429, 127 (1988).

[48] J. R. Forshaw and D. A. Ross, Quantum Chromodynamics and the Pomeron, Cambridge University Press, 1997.

[49] B. Ioffe, V. Fadin, L. Lipatov, Quantum Chromodynamics: Perturbative and Nonperturbative Aspects, Cambridge Monographs on Particle Physics, Nuclear Physics and Cosmology (2014).

[50] F. Wilczek, Fractional Statistics and Anyons Superconductivity, World Scientific 1990.

[51] M. S. Plyushchay, Nucl. Phys. B 589, (2000), 413.

[52] A. Bottino, A. Longoni, T. Regge, Il Nuovo Cimento 23 (1962), 954.

[53] S. Mandelstam, Ann. of Phys. 19, 254 (1959).

[54] R. Newton, Journ. of Math. Phys. 3 (1962), 867.

[55] E. Predazzi, T. Regge, Il Nuovo Cimento 24 (1962), 518.

[56] F. Calogero, Il Nuovo Cimento 28, 761 (1963).

[57] Ya.I. Azimov, arXiv:1307.2116, Sov. J. Nucl. Phys. 4 (1967) 469.

[58] R. L. Omnes, V. A. Alessandrini, Phys. Rev. 136, B 1137 (1964).

[59] M. Andrews, J. Gunson, Jour. of Math. Phys. 5, 1391 (1964).

[60] J. M. Charap, E. J. Squires, Ann. of Phys. 20, 145 (1962); ibid 21, 8 (1963).

[61] F. Calogero, J. Charap, E. Squires, Ann. of Phys. 25, 325 (1963).

[62] W. Drechsler, Il Nuovo Cimento 53 (1968), 115.

[63] M. Toller, Il Nuovo Cimento 37 (1965), 631; ibid 53 (1968), 671; ibid 54 (1968), 295.

[64] L. Sertorio, M. Toller, Il Nuovo Cimento 33 (1964), 413.

[65] G. Cosenza, A. Sciarrino, M. Toller, Il Nuovo Cimento 57 (1968), 253; ibid 62, 999.

[66] T. T. Wu, C. N. Yang, Phys. Rev. D 12 (1975) 3845.

[67] T. T. Wu, C. N. Yang, Nucl. Phys. B 107 (1976), 365.

[68] S. Mandelstam, Il Nuovo Cimento 30 (1963), 113; ibid. 30 (1963), 1127; ibid. 30 (1963), 1148.

[69] V. N. Gribov, Nucl. Phys. 22 (1961) 249; Sov. J. Nucl. Phys. 5 (1967) 138

[70] V. N. Gribov, I. A. Pomeranchuk, Phys. Lett. 2, 239 (1962). 
[71] M. Marino, Instantons and Large N: An Introduction to Non-Perturbative Methods in Quantum Field Theory, Cambridge University Press (2015).

[72] A. Bastai, L. Bertocchi, G. Furlan, M. Tonin, Il Nuovo Cimento 30 (1963), 1532.

[73] A. Bastai, L. Bertocchi, S. Fubini, G. Furlan, M. Tonin, Il Nuovo Cimento 30 (1963), 1512.

[74] R. Oehme, Il Nuovo Cimento 25 (1962), 183.

[75] J. Challifour, R. J. Eden, Journ. of Math. Phys. 4 (1963), 359.

[76] C. A. Lopez, Nucl. Phys. 76 ( 1966) 672.

[77] J. D. Bjorken, T. T. Wu, Phys. Rev. 130 (1963), 2566.

[78] H. Cornille, Il Nuovo Cimento 33 (1964), 434.

[79] G. F. Chew, Lecture notes at Les Houches summer school (1960) published in: Relations de dispersion et particules elementaires (Paris, 1961).

[80] G. F. Chew, S. C. Frautschi, Phys. Rev. Lett. 7, 394 (1962).

[81] E. G. Beltrametti, Il Nuovo Cimento 25 (1962), 1393.

[82] A. Donnachie, P. V. Landshoff, Phys. Lett. B 207 (1988) 319; Nucl. Phys. B 311 (1989) 509; Phys. Lett. B 296 (1992) 227.

[83] Particles Data Group, see the link: pdg.lbl.gov/2015/reviews/rpp2015-rev-cross-section-plots.pdf

[84] P. Skands, Soft QCD: Theory, Boston Jets Workshop MIT, January 2014.

[85] G. 't Hooft, Int. Conf. on High Energy Physics, Palermo (1975); Nucl. Phys. B 190 ( 1981) 455. 